\title{
2010 Idaho National Laboratory Water Use Report and Comprehensive Well Inventory (Revision 19)
}

June 2011

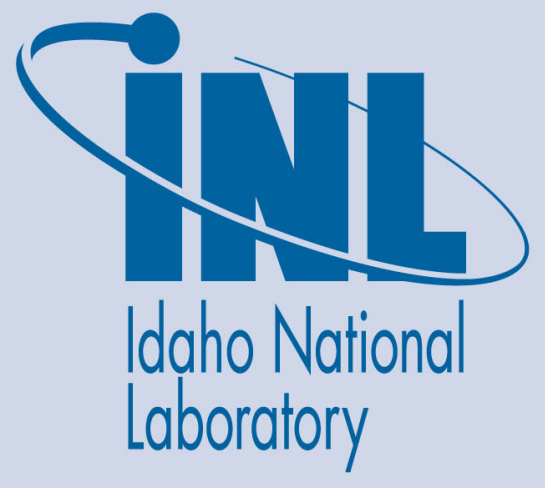

The INL is a U.S. Department of Energy National Laboratory operated by Battelle Energy Alliance 


\section{DISCLAIMER}

This information was prepared as an account of work sponsored by an agency of the U.S. Government. Neither the U.S. Government nor any agency thereof, nor any of their employees, makes any warranty, expressed or implied, or assumes any legal liability or responsibility for the accuracy, completeness, or usefulness, of any information, apparatus, product, or process disclosed, or represents that its use would not infringe privately owned rights. References herein to any specific commercial product, process, or service by trade name, trade mark, manufacturer, or otherwise, does not necessarily constitute or imply its endorsement, recommendation, or favoring by the U.S. Government or any agency thereof. The views and opinions of authors expressed herein do not necessarily state or reflect those of the U.S. Government or any agency thereof. 
INL/EXT-11-21880

\section{Idaho National Laboratory Water Use Report and Comprehensive Well Inventory (Revision 19)}

June 2011

Idaho National Laboratory Idaho Falls, Idaho 83415

http:www.inl.gov

Prepared for the

U.S. Department of Energy

Office of Nuclear Energy

Under DOE Idaho Operations Office

Contract DE-AC07-05ID14517 



\begin{abstract}
This 2010 Idaho National Laboratory Water Use Report and Comprehensive Well Inventory (Revision 19) provides water use information (monthly annual average and total annual volume) for production and potable water wells at the Idaho National Laboratory for Calendar Year 2010. It also provides detailed information for new, modified, and abandoned (decommissioned) wells and holes. Five new wells were drilled and completed in the latter part of Calendar Years 2009 and 2010. Two wells were modified in Calendar Year 2010 and 66 wells and boreholes reported as abandoned (decommissioned). Detailed construction information for the new and modified wells, along with abandonment information for older wells, is provided. Location maps are provided if survey information was available.

This report is being submitted in accordance with the Water Rights Agreement between the State of Idaho and the United States, for the United States Department of Energy (dated 1990) and the subsequent Partial Decree for Water Right 34-10901 issued June 20, 2003.
\end{abstract}




\section{CONTENTS}

ABSTRACT

ACRONYMS ix

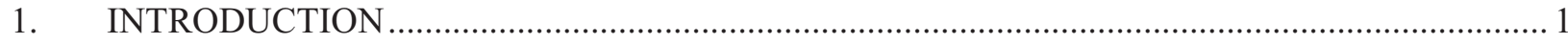

2. 2010 WATER USE INFORMATION FOR THE IDAHO NATIONAL LABORATORY .............. 2

2.1 Water Volume for Individual Idaho National Laboratory Production or Potable

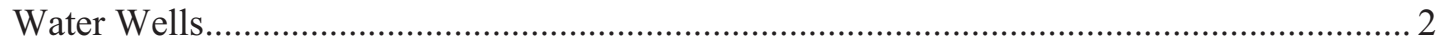

2.2 Combined Total Volume Diverted from All Production and Potable Water Wells................. 7

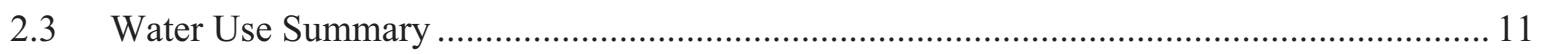

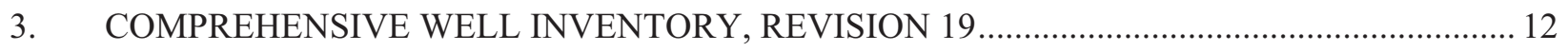

3.1 Idaho National Laboratory New and Modified Wells in Calendar Year 2010 .................... 12

3.2 Idaho National Laboratory Wells Decommissioned in CY 2010 ...................................... 15

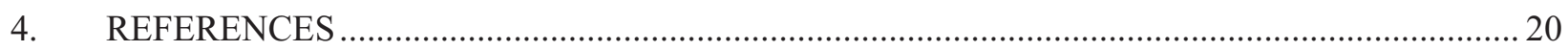

Appendix A-Maps and Construction Diagrams for New and Modified Wells ..................................... 21

Appendix B-Maps and Construction Diagrams for Abandoned Wells ............................................... 45

\section{FIGURES}

Figure A-1. Construction diagram for new well NRF-1 23

Figure A-2. Construction diagram for new well NRF-16 26

Figure A-3. Map showing location of new wells at the Idaho Nuclear Technology and Engineering Center

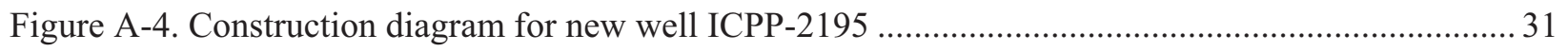

Figure A-5. Construction diagram for new well ICPP-2196 ........................................................... 32

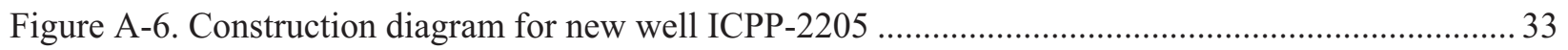

Figure A-7. Map showing location of modified well TRA-08 …........................................................ 35

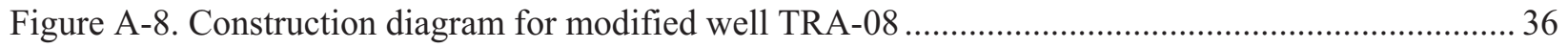

Figure A-9. Map showing location of modified well USGS-108 …...................................................... 37

Figure A-10. Construction diagram for modified well USGS-108 ….................................................... 39

Figure B-1. Map showing location of abandoned wells at the Idaho Nuclear Technology and Engineering Center 
Figure B-2. Construction diagram showing abandonment of USGS-050

Figure B-3. Map showing location of abandoned wells at Loss of Fluid Test Facility (LOFT) Complex

Figure B-4. Construction diagram showing abandonment of LOFT-A01 …........................................ 50

Figure B-5. Construction diagram showing abandonment of LOFT-A02 …...................................... 51

Figure B-6. Construction diagram showing abandonment of LOFT-A03 …...................................... 52

Figure B-7. Construction diagram showing abandonment of LOFT-A04 ............................................. 53

Figure B-8. Construction diagram showing abandonment of LOFT-A05 …........................................ 54

Figure B-9. Construction diagram showing abandonment of LOFT-A06 …........................................ 55

Figure B-10. Construction diagram showing abandonment of LOFT-A07 …....................................... 56

Figure B-11. Construction diagram showing abandonment of LOFT-A08 …....................................... 57

Figure B-12. Construction diagram showing abandonment of LOFT-A09 …........................................ 58

Figure B-13. Construction diagram showing abandonment of LOFT-A10 ….......................................59

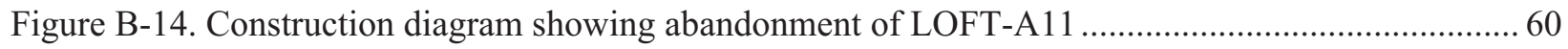

Figure B-15. Construction diagram showing abandonment of LOFT-A12 ….................................... 61

Figure B-16. Construction diagram showing abandonment of LOFT-A13 …..................................... 62

Figure B-17. Construction diagram showing abandonment of LOFT-A14 …..................................... 63

Figure B-18. Construction diagram showing abandonment of LOFT-A15 …...................................... 64

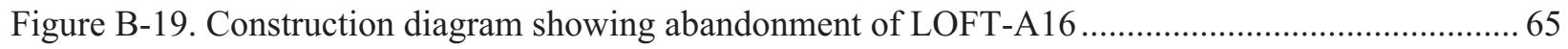

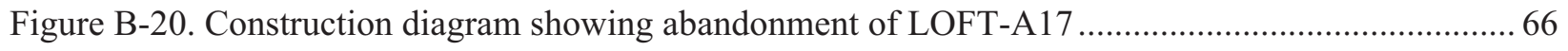

Figure B-21. Construction diagram showing abandonment of LOFT-A18 …................................... 67

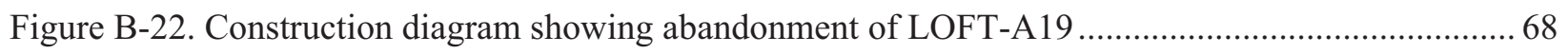

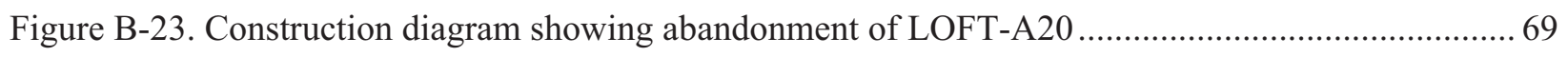

Figure B-24. Map showing locations of abandoned wells and holes at Advanced Test Reactor

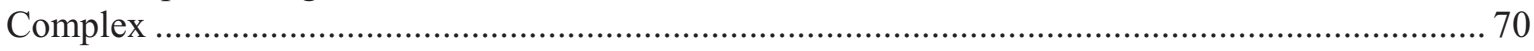

Figure B-25. Construction diagram showing abandonment of SB-01 ............................................. 71

Figure B-26. Construction diagram showing abandonment of SB-02 …............................................ 72 


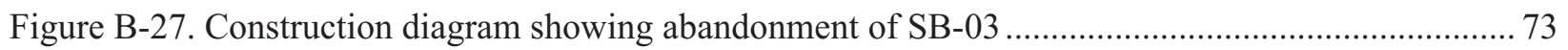

Figure B-28. Construction diagram showing abandonment of SB-04 .............................................. 74

Figure B-29. Construction diagram showing abandonment of SB-05 ............................................... 75

Figure B-30. Construction diagram showing abandonment of SB-06 ….............................................. 76

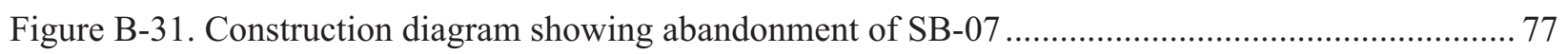

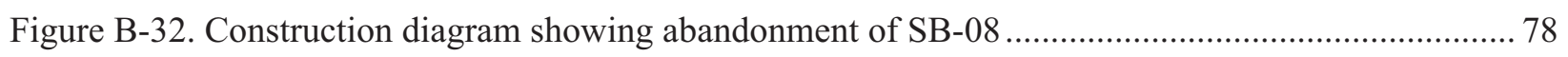

Figure B-33. Construction diagram showing abandonment of TRA-06 ............................................... 79

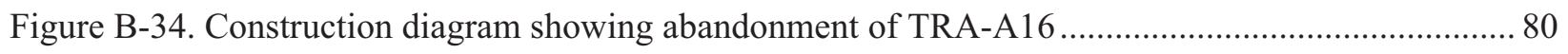

Figure B-35. Construction diagram showing abandonment of TRA-A17 …...................................... 81

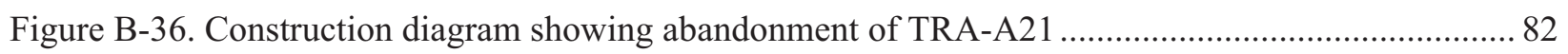

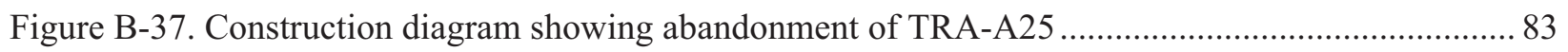

Figure B-38. Construction diagram showing abandonment of TRA-A27 .......................................... 84

Figure B-39. Construction diagram showing abandonment of TRA-A28 …...................................... 85

Figure B-40. Construction diagram showing abandonment of TRA-A29 …..................................... 86

Figure B-41. Construction diagram showing abandonment of TRA-A30 …....................................... 87

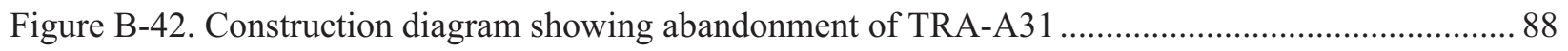

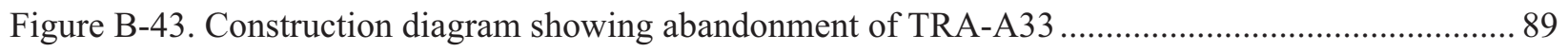

Figure B-44. Construction diagram showing abandonment of TRA-A34 …........................................ 90

Figure B-45. Construction diagram showing abandonment of TRA-A35 …....................................... 91

Figure B-46. Construction diagram showing abandonment of TRA-A37 ........................................... 92

Figure B-47. Construction diagram showing abandonment of TRA-A38 …....................................... 93

Figure B-48. Construction diagram showing abandonment of TRA-A39 …........................................ 94

Figure B-49. Construction diagram showing abandonment of TRA-A49 …....................................... 95

Figure B-50. Construction diagram showing abandonment of TRA-A51 ........................................... 96

Figure B-51. Construction diagram showing abandonment of TRA-A58 …........................................ 97

Figure B-52. Construction diagram showing abandonment of TRA-A76 ........................................... 98 
Figure B-53. Construction diagram showing abandonment of TRA-A77.

Figure B-54. Map showing locations of abandoned wells at the Radioactive Waste Management Complex

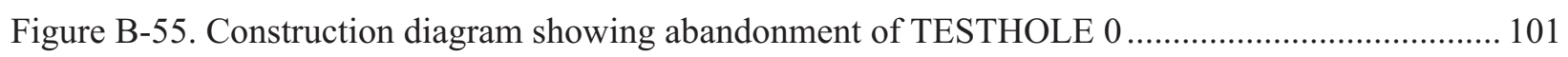

Figure B-56. Construction diagram showing abandonment of W-01 ….......................................... 102

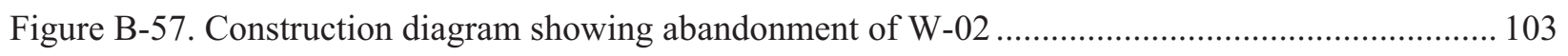

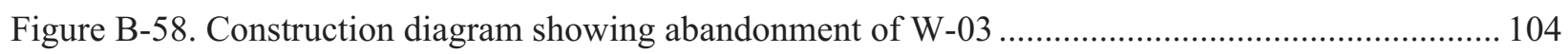

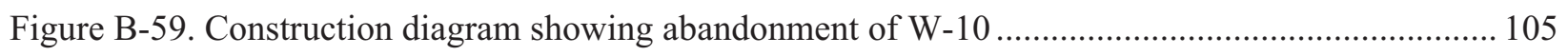

Figure B-60. Map showing abandoned well "BORAX" near the Radioactive Waste Management

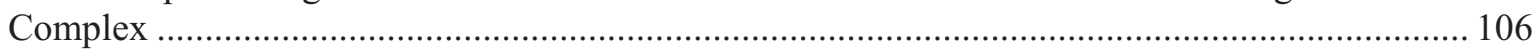

Figure B-61. Construction diagram showing abandonment of BORAX …......................................... 107

\section{TABLES}

Table 1. Advanced Test Reactor Complex water volume for 2010 ...................................................... 3

Table 2. Central Facilities Area water volume for 2010 ...................................................................... 3

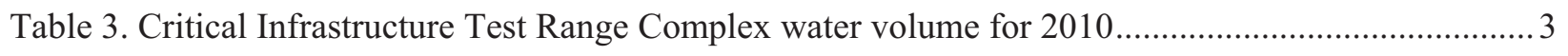

Table 4. Idaho Nuclear Technology and Engineering Center water volume for 2010 .............................. 4

Table 5. Materials and Fuels Complex water volume for 2010 ............................................................. 4

Table 6. Naval Reactors Facility water volume for 2010 .................................................................... 5

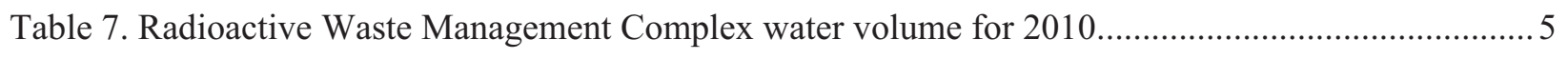

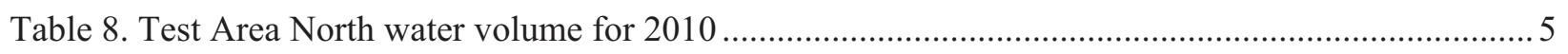

Table 9. Idaho National Laboratory water volume totals for 2010 ....................................................... 9

Table 10. Idaho National Laboratory new wells and modified wells in Calendar Year 2010 ................... 14

Table 11. Idaho National Laboratory boreholes and wells abandoned in Calendar Year 2010 ................. 17 


\section{ACRONYMS}

ATR Complex Advanced Test Reactor Complex

bls below land surface

CERCLA Comprehensive Environmental Response, Compensation, and Liability Act

CFA Central Facilities Area

CITRC Critical Infrastructure Test Range Complex

CWI Comprehensive Well Inventory

CY calendar year

IDWR Idaho Department of Water Resources

INL Idaho National Laboratory

INTEC Idaho Nuclear Technology and Engineering Center

LOFT Loss of Fluid Test Facility

MFC Materials and Fuels Complex

NRF Naval Reactors Facility

RWMC Radioactive Waste Management Complex

TAN Test Area North

USGS United States Geological Survey 


\section{Idaho National Laboratory Water Use Report and Comprehensive Well Inventory (Revision 19)}

\section{INTRODUCTION}

This 2010 Idaho National Laboratory Water Use Report and Comprehensive Well Inventory, (Revision 19) is being submitted in accordance with the Water Rights Agreement between the State of Idaho and the United States, for the United States Department of Energy (Department of Justice 1990) and the subsequent Partial Decree for Water Right 34-10901 (District Court 2003) issued June 20, 2003. As previously agreed (Street 2001), the annual Water Use Report and Comprehensive Well Inventory (CWI) are being combined and submitted as one report.

Section 2 provides water use information required by the Water Rights Agreement for production and potable water wells at the Idaho National Laboratory (INL) Site. The INL Site water use reported here is for Calendar Year (CY) 2010. Section 2.1 provides monthly annual average and total annual volume for each production or potable water well. Section 2.2 provides the monthly diversion rates and the total annual volume for all production or potable water wells.

Section 3 discusses Revision 19 to the CWI for the INL Site. Section 3.1 provides information for new and modified wells, and Section 3.2 provides information for abandoned (decommissioned) wells and boreholes. Five new wells were drilled and completed in the latter part of CY 2009 and CY 2010 and are included in this report. Two wells were modified in CY 2010. Section 3.2 identifies 66 wells and boreholes reported as abandoned (decommissioned). Appendix A provides detailed construction information for the new and modified wells. Appendix B contains maps showing the locations of abandoned wells and boreholes and diagrams, if available, that provide detailed construction and abandonment information. 


\section{2010 WATER USE INFORMATION FOR THE IDAHO NATIONAL LABORATORY}

\subsection{Water Volume for Individual Idaho National Laboratory Production or Potable Water Wells}

Eight major facilities are located at the INL Site:

- Advanced Test Reactor Complex (ATR Complex)

- Central Facilities Area (CFA)

- Critical Infrastructure Test Range Complex (CITRC)

- Idaho Nuclear Technology and Engineering Center (INTEC)

- Materials and Fuels Complex (MFC)

- Naval Reactors Facility (NRF)

- Radioactive Waste Management Complex (RWMC)

- $\quad$ Test Area North (TAN).

Each major facility is serviced by one or more production and/or potable water wells. Tables 1 through 8 show water information for production or potable wells at these facilities.

Seven wells are grouped under the CFA facility. Wells CFA-1 and CFA-2 serve the actual CFA. The other five wells (Badging Facility Well, EBR-1, Fire Station Well, Rifle Range Well, and Site-04 [Dairy Farm]) serve smaller facilities or processes. The Fire Station Well occasionally has been used for filling water trucks for construction purposes. However, for 2010, the Fire Station Well was not used because the pump was inoperable. The Dairy Farm Well is used for irrigating various research projects. The wells identified at other INL Site facilities provide water primarily for that specific facility.

Each table provides the monthly annual average and total annual volume of water diverted from each production or potable well during CY 2010. Each well is identified by its official well name, the most common alias name, and the well identification number. Footnotes are provided where applicable. 


\begin{tabular}{|c|c|c|c|c|c|c|c|c|c|c|c|c|c|c|c|c|}
\hline \multicolumn{17}{|c|}{$\begin{array}{l}\text { Volume in } \\
\text { Gallons }\end{array}$} \\
\hline Well & Alias & Well ID & January & February & March & April & May & June & July & August & September & October & November & December & $\begin{array}{l}\text { Total Annual } \\
\text { Volume }\end{array}$ & $\begin{array}{l}\text { Average Monthly } \\
\text { Volume }\end{array}$ \\
\hline TRA-01 & NO. 1 DEEP WELL & 356 & $28,867,000$ & $27,608,000$ & $34,283,000$ & $26,078,000$ & $14,027,000$ & $3,198,000$ & $30,408,000$ & $98,000^{\mathrm{a}}$ & $38,463,000$ & $17,813,000$ & $39,881,000$ & $10,528,000$ & $271,252,000$ & $22,604,333$ \\
\hline TRA-03 & NO. 3 DEEP WELL & 358 & 524,000 & 30,000 & 29,000 & 398,000 & 21,000 & 645,000 & 23,000 & 591,000 & 27,000 & $1,924,700$ & 7,100 & 1,000 & $4,220,800$ & 351,733 \\
\hline TRA-04 & NO. 4 DEEP WELL & 359 & $1,116,000$ & 0 & 795,000 & 644,000 & $25,319,000$ & $27,414,000$ & $8,833,000$ & $47,806,000$ & $6,698,000$ & $23,905,000$ & 0 & $23,322,000$ & $165,852,000$ & $13,821,000$ \\
\hline TRA-1863 & & 1863 & $2,697,200$ & $2,651,200$ & $2,562,100$ & $2,709,500$ & $2,611,500$ & $3,272,400$ & $3,563,100$ & $3,270,900$ & $2,896,500$ & $3,012,100$ & $2,392,600$ & $2,926,700$ & $34,565,800$ & $2,880,483$ \\
\hline \multicolumn{3}{|c|}{ Monthly total } & $33,204,200$ & $30,289,200$ & $37,669,100$ & $29,829,500$ & $41,978,500$ & $34,529,400$ & $42,827,100$ & $51,765,900$ & $48,084,500$ & $46,654,800$ & $42,280,700$ & $36,777,700$ & & \\
\hline \multicolumn{17}{|c|}{ Total annual volume for ATR Complex: $\mathbf{4 7 5 , 8 9 0 , 6 0 0}$} \\
\hline \multicolumn{17}{|c|}{ a. TRA-01 total flow for August is calculated based on hours of operation due to flow totalizer being out of service. } \\
\hline \multirow{2}{*}{\multicolumn{17}{|c|}{$\begin{array}{l}\text { Depth to water, static water level: } \\
\text { Date }\end{array}$}} \\
\hline$\underline{\mathrm{D}}$ & & TRA-1863 & & & & & & & & & & & & & & \\
\hline \multicolumn{17}{|c|}{ September 30, 2010} \\
\hline
\end{tabular}

Table 2. Central Facilities Area water volume for 2010.

\begin{tabular}{|c|c|c|c|c|c|c|c|c|c|c|c|c|c|c|c|c|}
\hline \multicolumn{17}{|c|}{$\begin{array}{l}\text { Volume in } \\
\text { Gallons }\end{array}$} \\
\hline Well & Alias & Well ID & January & February & March & April & May & June & July & August & September & October & November & December & $\begin{array}{l}\text { Total Annual } \\
\text { Volume }\end{array}$ & $\begin{array}{c}\text { Average } \\
\text { Monthly Volume }\end{array}$ \\
\hline CFA-1 & CFA-651 & 93 & $1,299,100$ & $1,821,700$ & $1,520,400$ & $1,199,800$ & $1,077,300$ & $1,070,800$ & $5,525,600$ & $3,996,100$ & $4,098,400$ & $1,755,100$ & $1,412,300$ & $1,439,200$ & $26,215,800$ & $2,184,650$ \\
\hline CFA-2 & CFA-642 & 94 & 304,600 & 2,700 & 300,300 & 841,700 & $3,598,100$ & $5,034,100$ & $5,557,700$ & $7,721,100$ & 0 & 0 & 0 & 0 & $23,360,300$ & $1,946,692$ \\
\hline \begin{tabular}{|l} 
Badging Facility Well \\
\end{tabular} & B27-605 Main Gate & 88 & 7,920 & 14,250 & 29,890 & 3,770 & 3,770 & 5,340 & 3,630 & 11,210 & 4,620 & 3,350 & 15,670 & 9,140 & 112,560 & 9,380 \\
\hline EBR-1 & & 149 & 494 & 356 & 965 & 1,119 & 2,684 & 4,910 & 7,841 & 9,783 & 1,267 & 1,167 & 1,462 & 809 & 32,857 & 2,738 \\
\hline Rifle Range Well & B21-607 Gun Range & 267 & 1,280 & 1,380 & 2,120 & 2,090 & 1,530 & 6,980 & 1,710 & 2,660 & 2,970 & 2,620 & 1,970 & 1,020 & 28,330 & 2,361 \\
\hline Site-04 & B16-604 Dairy Farm & 273 & 0 & 0 & 0 & 41,050 & 0 & 21,920 & 20,730 & 0 & 0 & 0 & 0 & 0 & 83,700 & 6,975 \\
\hline Fire Station Well & Fire Station \#2 & 158 & 0 & 0 & 0 & 0 & 0 & 0 & 0 & 0 & 0 & 0 & 0 & 0 & 0 & 0 \\
\hline \multicolumn{3}{|l|}{ Monthly total } & $1,613,394$ & $1,840,386$ & $1,853,675$ & $2,089,529$ & $4,683,384$ & $6,144,050$ & $11,117,211$ & $11,740,853$ & $4,107,257$ & $1,762,237$ & $1,431,402$ & $1,450,169$ & & \\
\hline
\end{tabular}

Table 3. Critical Infrastructure Test Range Complex water volume for 2010.

\begin{tabular}{|c|c|c|c|c|c|c|c|c|c|c|c|c|c|c|c|c|}
\hline \multicolumn{17}{|c|}{$\begin{array}{c}\text { Volume in } \\
\text { Gallons }\end{array}$} \\
\hline Well & Alias & Well ID & January & February & March & April & May & June & July & August & September & October & November & December & $\begin{array}{c}\text { Total Annual } \\
\text { Volume }\end{array}$ & $\begin{array}{l}\text { Average Monthly } \\
\text { Volume }\end{array}$ \\
\hline SPERT-1 & PBF Deep Well No. 1 & 280 & 304,600 & 229,800 & 108,900 & 157,500 & 82,900 & 71,200 & 161,500 & 71,400 & 121,000 & 130,300 & 59,100 & 65,500 & $1,563,700$ & 130,308 \\
\hline SPERT-2 & PBF Deep Well No. 2 & 281 & 142,800 & 250,300 & 344,200 & 178,200 & 108,500 & 98,000 & 59,500 & 58,700 & 107,200 & 23,500 & 36,800 & 72,500 & $1,480,200$ & 123,350 \\
\hline \multicolumn{3}{|c|}{ Monthly total } & 447,400 & 480,100 & 453,100 & 335,700 & 191,400 & 169,200 & 221,000 & 130,100 & 228,200 & 153,800 & 95,900 & 138,000 & & \\
\hline
\end{tabular}


Table 4. Idaho Nuclear Technology and Engineering Center water volume for 2010

\begin{tabular}{|c|c|c|c|c|c|c|c|c|c|c|c|c|c|c|c|c|}
\hline \multicolumn{17}{|c|}{$\begin{array}{l}\text { Volume in } \\
\text { Gallons }\end{array}$} \\
\hline Well & Alias & Well ID & January & February & March & April & May & June & July & August & September & October & November & December & $\begin{array}{c}\text { Total Annual } \\
\text { Volume }\end{array}$ & $\begin{array}{l}\text { Average } \\
\text { Monthly } \\
\text { Volume }\end{array}$ \\
\hline CPP- $01^{a}$ & F-UTI-670 & 98 & 641,000 & $12,404,000$ & 418,000 & $23,063,000$ & $2,930,000$ & $19,196,000$ & $14,162,000$ & $10,614,000$ & 376,000 & $14,841,000$ & 163,000 & $12,576,000$ & $111,384,000$ & $9,282,000$ \\
\hline CPP- $02^{a, b}$ & F-UTI-671 & 99 & $18,103,000$ & 4,000 & $28,537,000$ & 676,000 & $18,841,000$ & 0 & $2,219,000$ & 0 & $11,111,000$ & 585,000 & $12,238,000$ & 0 & $92,314,000$ & $7,692,833$ \\
\hline CPP- $04^{\circ}$ & & 101 & 649,668 & 522,708 & 413,602 & 290,500 & 286,801 & 304,739 & 317,266 & 408,943 & 314,936 & 271,029 & 203,577 & 154,401 & $4,138,167$ & 344,847 \\
\hline ICPP-POT-A-012 ${ }^{\circ}$ & $\begin{array}{l}\text { F-UTI-699 or } \\
\text { CPP-05 }\end{array}$ & 1186 & 649,668 & 522,708 & 413,602 & 290,500 & 286,801 & 304,739 & 317,266 & 408,943 & 314,936 & 271,029 & 203,577 & 154,401 & $4,138,167$ & 344,847 \\
\hline \multicolumn{3}{|l|}{ Monthly total } & $20,043,335$ & $13,453,416$ & $29,782,203$ & $24,320,000$ & $22,344,601$ & $19,805,478$ & $17,015,531$ & $11,431,885$ & $12,116,871$ & $15,968,058$ & $12,808,154$ & $12,884,801$ & & \\
\hline \multicolumn{17}{|c|}{ Total annual volume for INTEC: $\mathbf{2 1 1 , 9 7 4 , \mathbf { 3 3 3 }}$} \\
\hline
\end{tabular}

Table 5. Materials and Fuels Complex water volume for 2010

\begin{tabular}{|c|c|c|c|c|c|c|c|c|c|c|c|c|c|c|c|c|}
\hline \multicolumn{17}{|c|}{$\begin{array}{l}\text { Volume in } \\
\text { Gallons }\end{array}$} \\
\hline Well & Alias & Well ID & January & February & March & April & May & June & July & August & September & October & November & December & $\begin{array}{l}\text { Total Annual } \\
\text { Volume }\end{array}$ & $\begin{array}{c}\text { Average Monthly } \\
\text { Volume }\end{array}$ \\
\hline EBR-II \#1 $1^{\mathrm{a}, \mathrm{b}}$ & EBR-1 & 150 & 0 & 0 & 0 & 0 & 0 & 779,000 & $1,178,500$ & $1,094,500$ & 543,500 & 641,000 & 621,000 & 648,000 & $5,505,500$ & 458,792 \\
\hline EBR-II $\# 2^{b}$ & EBR-II & 151 & $2,196,000$ & $1,576,000$ & $1,655,000$ & $1,822,000$ & $1,891,000$ & 779,000 & $1,178,500$ & $1,094,500$ & 543,500 & 641,000 & 621,000 & 648,000 & $14,645,500$ & $1,220,458$ \\
\hline \multicolumn{3}{|l|}{ Monthly total } & $2,196,000$ & $1,576,000$ & $1,655,000$ & $1,822,000$ & $1,891,000$ & $1,558,000$ & $2,357,000$ & $2,189,000$ & $1,087,000$ & $1,282,000$ & $1,242,000$ & $1,296,000$ & & \\
\hline \multicolumn{17}{|c|}{ Total annual volume for MFC: $\mathbf{2 0 , 1 5 1 , 0 0 0}$} \\
\hline \multicolumn{17}{|c|}{$\begin{array}{l}\text { a. Well EBR-II \#1 was removed from service beginning in August } 2009 \text { for upgrade of the electrical system, and was placed back in service in June } 2010 \text {. } \\
\text { b. The two wells share one flow meter. Operations switch between the wells, so the totals are estimated to be } 50 \% \text { for each well from June through December. }\end{array}$} \\
\hline \multicolumn{17}{|c|}{ Depth to water, static water level: } \\
\hline Date & $\underline{E B R-I I \# 1}$ & $\underline{E B R-I I H Z}$ & & & & & & & & & & & & & & \\
\hline May 2010 & Not measured & 658.5 feet bl. & & & & & & & & & & & & & & \\
\hline November 2010 & 659.5 feet bls & 660.0 feet bl: & & & & & & & & & & & & & & \\
\hline
\end{tabular}


Table 6. Naval Reactors Facility water volume for 2010.

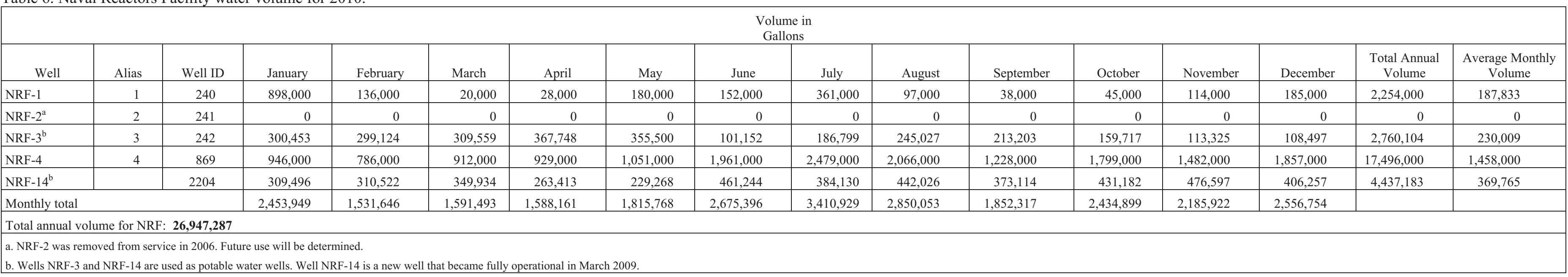

Table 7. Radioactive Waste Management Complex water volume for 2010

\begin{tabular}{|c|c|c|c|c|c|c|c|c|c|c|c|c|c|c|c|c|}
\hline \multicolumn{17}{|c|}{$\begin{array}{c}\text { Volume in } \\
\text { Gallons }\end{array}$} \\
\hline Well & Alias & Well ID & January & February & March & April & May & June & July & August & September & October & November & December & \begin{tabular}{|c|} 
Total Annual \\
Volume
\end{tabular} & \begin{tabular}{|l} 
Average Monthly \\
Volume
\end{tabular} \\
\hline RWMC Production & & 268 & 774,200 & 654,500 & 882,200 & 744,900 & 650,100 & $1,023,900$ & $1,373,500$ & $1,492,300$ & $1,203,300$ & 858,700 & 444,400 & 435,600 & \begin{tabular}{|l}
$10,537,600$ \\
\end{tabular} & 878,133 \\
\hline PIT 9 Production Well & & 2155 & 0 & 0 & 0 & 0 & 0 & 0 & 0 & 0 & 158,700 & 361,100 & 53,200 & 0 & 573,000 & 47,750 \\
\hline Monthly total & & & 774,200 & 654,500 & 882,200 & 744,900 & 650,100 & $1,023,900$ & $1,373,500$ & $1,492,300$ & $1,362,000$ & $1,219,800$ & 497,600 & 435,600 & & \\
\hline
\end{tabular}

Table 8. Test Area North water volume for 2010.

\begin{tabular}{|c|c|c|c|c|c|c|c|c|c|c|c|c|c|c|c|c|}
\hline \multicolumn{17}{|c|}{$\begin{array}{l}\text { Volume in } \\
\text { Gallons }\end{array}$} \\
\hline Well & Alias & Well ID & January & February & March & April & May & June & July & August & September & October & November & December & $\begin{array}{l}\text { Total Annual } \\
\text { Volume }\end{array}$ & $\begin{array}{l}\text { Average Monthly } \\
\text { Volume }\end{array}$ \\
\hline ANP- $01^{a}$ & TAN-612 & 69 & 0 & 0 & 0 & 0 & 0 & 0 & 0 & 0 & 0 & 0 & 0 & 0 & 0 & 0 \\
\hline ANP-02 & TAN-613 & 70 & 181,600 & 172,700 & 169,800 & 161,000 & 101,900 & 137,300 & 116,200 & 139,800 & 68,900 & 156,200 & 62,700 & 70,300 & $1,538,400$ & 128,200 \\
\hline FET-1 & TAN-632 & 154 & 145,400 & 150,600 & 153,900 & 106,300 & 93,700 & 49,500 & 54,000 & 493,200 & 328,500 & 482,900 & 160,300 & 107,600 & $2,325,900$ & 193,825 \\
\hline FET-2 & TAN-639 & 155 & 207,700 & 160,000 & 222,100 & 274,800 & 535,300 & 799,200 & $1,219,700$ & 565,800 & 407,100 & 281,000 & 173,700 & 167,400 & $5,013,800$ & 417,817 \\
\hline \multicolumn{3}{|c|}{ Monthly total } & 534,700 & 483,300 & 545,800 & 542,100 & 730,900 & 986,000 & $1,389,900$ & $1,198,800$ & 804,500 & 920,100 & 396,700 & 345,300 & & \\
\hline
\end{tabular}





\subsection{Combined Total Volume Diverted from All Production and Potable Water Wells}

Table 9 provides the combined total volume from all production and potable water wells at the INL Site during CY 2010. Table 9 includes:

- Total monthly volume of water diverted for each major INL Site facility

- The combined total monthly volume from all the major INL Site facilities

- Monthly average for all wells combined

- Total annual volume diverted at the INL Site. 


\begin{tabular}{|c|c|c|c|c|c|c|c|c|c|c|c|c|}
\hline \multicolumn{13}{|c|}{$\begin{array}{l}\text { Volume in } \\
\text { Gallons }\end{array}$} \\
\hline Facility & January & February & March & April & May & June & July & August & September & October & November & December \\
\hline $\begin{array}{l}\text { Advanced Test Reactor } \\
\text { Complex }\end{array}$ & $33,204,200$ & $30,289,200$ & $37,669,100$ & $29,829,500$ & $41,978,500$ & $34,529,400$ & $42,827,100$ & $51,765,900$ & $48,084,500$ & $46,654,800$ & $42,280,700$ & $36,777,700$ \\
\hline Central Facilities Area & $1,613,394$ & $1,840,386$ & $1,853,675$ & $2,089,529$ & $4,683,384$ & $6,144,050$ & $11,117,211$ & $11,740,853$ & $4,107,257$ & $1,762,237$ & $1,431,402$ & $1,450,169$ \\
\hline $\begin{array}{l}\text { Critical Infrastructure Test } \\
\text { Range Complex }\end{array}$ & 447,400 & 480,100 & 453,100 & 335,700 & 191,400 & 169,200 & 221,000 & 130,100 & 228,200 & 153,800 & 95,900 & 138,000 \\
\hline $\begin{array}{l}\text { Idaho Nuclear Technology } \\
\text { and Engineering Center }\end{array}$ & $20,043,335$ & $13,453,416$ & $29,782,203$ & $24,320,000$ & $22,344,601$ & $19,805,478$ & $17,015,531$ & $11,431,885$ & $12,116,871$ & $15,968,058$ & $12,808,154$ & $12,884,801$ \\
\hline $\begin{array}{l}\text { Materials and Fuels } \\
\text { Complex }\end{array}$ & $2,196,000$ & $1,576,000$ & $1,655,000$ & $1,822,000$ & $1,891,000$ & $1,558,000$ & $2,357,000$ & $2,189,000$ & $1,087,000$ & $1,282,000$ & $1,242,000$ & $1,296,000$ \\
\hline Naval Reactors Facility & $2,453,949$ & $1,531,646$ & $1,591,493$ & $1,588,161$ & $1,815,768$ & $2,675,396$ & $3,410,929$ & $2,850,053$ & $1,852,317$ & $2,434,899$ & $2,185,922$ & $2,556,754$ \\
\hline $\begin{array}{l}\text { Radioactive Waste } \\
\text { Management Complex }\end{array}$ & 774,200 & 654,500 & 882,200 & 744,900 & 650,100 & $1,023,900$ & $1,373,500$ & $1,492,300$ & $1,362,000$ & $1,219,800$ & 497,600 & 435,600 \\
\hline Test Area North & 534,700 & 483,300 & 545,800 & 542,100 & 730,900 & 986,000 & $1,389,900$ & $1,198,800$ & 804,500 & 920,100 & 396,700 & 345,300 \\
\hline Monthly total & $61,267,178$ & $50,308,548$ & $74,432,571$ & $61,271,890$ & $74,285,653$ & $66,891,424$ & $79,712,171$ & $82,798,891$ & $69,642,645$ & $70,395,694$ & $60,938,378$ & $55,884,324$ \\
\hline \multicolumn{2}{|c|}{ Maximum monthly diversion total } & \multicolumn{2}{|c|}{$82,798,891$ for August 10} & & & & & & & & & \\
\hline \multicolumn{2}{|c|}{ Total average monthly volume } & \multicolumn{2}{|c|}{$67,319,114$} & & & & & & & & & \\
\hline \multicolumn{2}{|l|}{ Total for 2010} & \multicolumn{2}{|c|}{$807,829,367$} & & & & & & & & & \\
\hline
\end{tabular}





\subsection{Water Use Summary}

The INL Site's Federal Reserved Water Right is 35,000 acre-ft per year $\left(1.14 \times 10^{10} \mathrm{gal} / \mathrm{yr}\right)$ and will not exceed a maximum diversion rate of $80 \mathrm{ft}^{3} / \mathrm{s}(35,904 \mathrm{gpm})$. The total volume of water diverted at the INL Site for CY 2010 was approximately $8.08 \times 10^{8}$ gal (see Table 9) or approximately $7.1 \%$ of the annual water right. The maximum diversion rate occurred during August at a rate of $4.1 \mathrm{ft}^{3} / \mathrm{s}(1,855 \mathrm{gpm})$. The average monthly volume of water diverted for all INL Site production and potable wells was approximately $6.73 \times 10^{7}$ gal. The INL's water use remained well within the established water right. 


\section{COMPREHENSIVE WELL INVENTORY, REVISION 19 \\ 3.1 Idaho National Laboratory New and Modified Wells in Calendar Year 2010}

Five new wells were constructed at the INL Site in CY 2010: NRF-15, NRF-16, ICPP-2195, ICPP-2196, and ICPP-2205. Two wells, TRA-08 and USGS-108, were deepened and modified. These additions and modifications are listed in Table 10.

Drilling for NRF-15 began in October 2008 by the United States Geological Survey (USGS) and was completed in May 2009. Total depth for this well was reached at 759 feet below land surface (bls). This well was intended to be a replacement monitoring well for NRF-13. Well NRF-15 was intended to be used as an upgradient monitoring well. However, initial sample results from NRF-15, collected in November and December of 2008, showed higher than expected concentrations of chloride and sulfate, indicating that the groundwater quality was being influenced by NRF operations. Therefore, NRF-15 was completed as a piezometer well, with one shallow (screened at 380 feet bls to 390 feet bls) and one deep (screened at 612 feet bls to 622 feet bls) completion (see Figure A-1).

A decision was made to move to a new location and drill the NRF-13 replacement monitoring well (NRF-16). Well NRF-16 is located approximately 1 mile north of NRF 15. Construction of NRF-16 began in August 2009, and was completed in November 2009, to a depth of 425 feet bls (see Figure A-2). Water sample results indicated NRF-16 would meet NRF needs for an upgradient monitoring well (DOE-ID 2010). Coordinates for NRF-15 and NRF-16 have not been verified; therefore, a map is not included.

Three new perched water monitoring wells, ICPP-2195, ICPP-2196, and ICPP-2205 (see Figure A-3), were installed as part of the Phase 1 post-closure period of the CPP-601/627/640 landfill closure in accordance with HWMA/RCRA Post-Closure Plan for the CPP-601/627/640 Landfill (DOE-ID 2009). The new perched monitoring wells are intended to measure the groundwater elevations and monitor the groundwater quality below the waste management area. Drilling for ICPP-2195 began on February 16, 2010, and was completed on February 25, 2010. The well was drilled to 121 feet bls and was completed to a depth of 107 feet bls (see Figure A-4). Drilling for ICPP-2196 began on March 1, 2010, and was completed on March 22, 2010. The well was drilled to 143 feet bls and completed to a depth of 142 feet bls (see Figure A-5). Drilling for ICPP-2205 began on March 23, 2010, and was completed on March 29, 2010. The well was drilled to 150 feet bls and was completed to a depth of 141 feet bls (see Figure A-6).

Well TRA-08 (see Figures A-7 and A-8) is used for both Comprehensive Environmental Response, Compensation, and Liability Act (CERCLA) and State of Idaho Wastewater Reuse Permit monitoring. The water volume in the well was not sufficient to allow purging of the well, which is required when sampling. The borehole diameter was increased at 405 feet bls from 4 inches to 6 inches, and the well was deepened to 531.5 feet bls. Slough material filled the borehole to 529.24 feet bls. The water level was at 488.8 feet bls on April 14, 2010. A 4-inch, stainless steel casing was set from -2.5 to 488.24 feet bls, and a stainless steel slotted screen was installed from 488.24 to 528.24 feet bls. A 5-hp pump was set at 519 feet bls, with the inlet at 522 feet bls (see Figure A-8). A new 12-inch-diameter, carbon steel protective casing was welded on at the surface. 
Well USGS-108 (see Figures A-9 and A-10) was originally drilled to 760 feet bls. In 2008, USGS-108 was cored from 760 feet bls to 1,218 feet bls to examine the lithology and establish zones of interest. In September 2010, the corehole was widened to a 4.75-inch borehole, and a Westbay multi-level monitoring system was installed (see Figure A-10). 
Table 10. Idaho National Laboratory new wells and modified wells in Calendar Year 2010.

\begin{tabular}{|c|c|c|c|c|c|c|c|c|}
\hline \multicolumn{9}{|c|}{ INL NEW WELLS AND MODIFICATIONS IN CY 2010} \\
\hline Well Name & Type & $\begin{array}{l}\text { Borehole } \\
\text { Depth } \\
\text { (ft bls) }\end{array}$ & $\begin{array}{l}\text { Casing Diameter } \\
\text { (in.) }\end{array}$ & $\begin{array}{c}\text { Construction } \\
\text { Material } \\
\end{array}$ & Status & Location & $\begin{array}{c}\text { Driller/ } \\
\text { License \# }\end{array}$ & Comments \\
\hline NRF-15 & $\begin{array}{l}\text { Observation/ } \\
\text { piezometer }\end{array}$ & 759 & $\begin{array}{l}10 \text { from } 0 \mathrm{ft}-16 \mathrm{ft} \\
4 \text { from }-1.5 \mathrm{ft}-128 \mathrm{ft} \\
1 \text { from }-2.13 \mathrm{ft}-622 \mathrm{ft} \\
(\text { screen from } 612 \mathrm{ft}-622 \mathrm{ft}) \\
1 \text { from }-2.03 \mathrm{ft}-390 \mathrm{ft} \\
(\text { screen from } 380 \mathrm{ft}-390 \mathrm{ft})\end{array}$ & $\begin{array}{l}\text { Carbon steel } \\
\text { Stainless steel }\end{array}$ & Active & $\begin{array}{l}\text { T04N, R30E, } \\
\text { Sec. 20, NW } 1 / 4, \\
\text { SW } 1 / 4, \text { NW } 1 / 4\end{array}$ & USGS & $\begin{array}{l}\text { Completed as a piezometer } \\
\text { well with a shallow and deep } \\
\text { monitoring interval. }\end{array}$ \\
\hline NRF-16 & Observation & 425 & $\begin{array}{l}10 \text { from } 0 \mathrm{ft}-15 \mathrm{ft} \\
7 \text { from }-2 \mathrm{ft}-339 \mathrm{ft} \\
5 \text { from } 302 \mathrm{ft}-422 \mathrm{ft} \\
(\text { screen from } 362 \mathrm{ft}-422 \mathrm{ft} \text { ) }\end{array}$ & $\begin{array}{l}\text { Carbon steel } \\
\text { Stainless steel }\end{array}$ & Active & $\begin{array}{l}\text { T04N, R30E, } \\
\text { Sec. 17, SW } 1 / 4, \\
\text { NW } 1 / 4, \text { SW } 1 / 4\end{array}$ & USGS & $\begin{array}{l}\text { CERCLA well. Upgradient } \\
\text { monitoring well. }\end{array}$ \\
\hline ICPP-2195 & Monitoring & 121 & $\begin{array}{l}6 \text { from }-3.7 \mathrm{ft}-56 \mathrm{ft} \\
2 \text { from }-3 \mathrm{ft}-107 \mathrm{ft} \\
(\text { screen from } 87 \mathrm{ft}-107 \mathrm{ft} \text { ) }\end{array}$ & $\begin{array}{l}\text { Carbon steel } \\
\text { Stainless Steel }\end{array}$ & Active & $\begin{array}{l}\text { T03N, R30E, } \\
\text { Sec. 19, NW } 1 / 4, \\
\text { NW 1/4, SW } 1 / 4\end{array}$ & $\begin{array}{l}\text { Major } \\
\text { Drilling Co./ } \\
\text { \#397 }\end{array}$ & $\begin{array}{l}\text { RCRA/CERCLA monitoring } \\
\text { well. }\end{array}$ \\
\hline ICPP-2196 & Monitoring & 143 & $\begin{array}{l}6 \text { from }-2.7 \mathrm{ft}-58 \mathrm{ft} \\
2 \text { from }-2.08 \mathrm{ft}-142 \mathrm{ft} \\
(\text { screen from } 117 \mathrm{ft}-142 \mathrm{ft})\end{array}$ & $\begin{array}{l}\text { Carbon steel } \\
\text { PVC }\end{array}$ & Active & $\begin{array}{l}\text { T03N, R30E, } \\
\text { Sec. } 19, \text { NW } 1 / 4, \\
\text { NW } 1 / 4, \text { SW } 1 / 4\end{array}$ & $\begin{array}{l}\text { Major } \\
\text { Drilling Co./ } \\
\# 397\end{array}$ & $\begin{array}{l}\text { RCRA/CERCLA monitoring } \\
\text { well. }\end{array}$ \\
\hline ICPP-2205 & Monitoring & 150 & $\begin{array}{l}6 \text { from }-3.7 \mathrm{ft}-56.5 \mathrm{ft} \\
2 \text { from }-3.4 \mathrm{ft}-141 \mathrm{ft} \\
(\text { screen from } 121 \mathrm{ft}-141 \mathrm{ft})\end{array}$ & Stainless steel & Active & $\begin{array}{l}\text { T03N, R30E, } \\
\text { Sec. } 19, \text { NW } 1 / 4, \\
\text { NW } 1 / 4, \text { SW } 1 / 4\end{array}$ & $\begin{array}{l}\text { Major } \\
\text { Drilling Co./ } \\
\# 397\end{array}$ & $\begin{array}{l}\text { RCRA/CERCLA monitoring } \\
\text { well. }\end{array}$ \\
\hline TRA-08 & Monitoring & 531.5 & $\begin{array}{l}8 \text { from } 0 \mathrm{ft}-70 \mathrm{ft} \\
6 \text { from }-1.78 \mathrm{ft}-405 \mathrm{ft} \\
4 \text { from }-2.52 \mathrm{ft}-528.24 \mathrm{ft} \\
(\text { screen from } 488.24 \mathrm{ft}- \\
528.24 \mathrm{ft})\end{array}$ & $\begin{array}{l}\text { Carbon steel } \\
\text { stainless steel }\end{array}$ & Active & $\begin{array}{l}\text { T03N, R29E, } \\
\text { Sec. } 23 \text { NE } 1 / 4, \\
\text { SE } 1 / 4, \text { NW } 1 / 4\end{array}$ & $\begin{array}{l}\text { Major } \\
\text { Drilling Co./ } \\
\text { \#397 }\end{array}$ & $\begin{array}{l}\text { CERCLA/Wastewater Reuse } \\
\text { well. }\end{array}$ \\
\hline USGS-108 & Monitoring & 1,218 & $\begin{array}{l}8 \text { from } 0 \mathrm{ft}-400 \mathrm{ft} \\
5 \text { from }-1 \mathrm{ft}-760 \mathrm{ft} \\
3 \text { from }-2.42 \mathrm{ft}-1,194 \mathrm{ft}\end{array}$ & $\begin{array}{l}\text { Carbon steel } \\
\text { PVC }\end{array}$ & Active & $\begin{array}{l}\text { T02N, R29E, } \\
\text { Sec. 35, SW } 1 / 4, \\
\text { SW } 1 / 4, \text { SW } 1 / 4\end{array}$ & USGS & $\begin{array}{l}\text { Widened to } 4.75 \text { in. from } \\
760 \mathrm{ft}-1,218 \mathrm{ft} \text {. Installed } \\
\text { Westbay multi-level } \\
\text { monitoring system. }\end{array}$ \\
\hline $\begin{array}{l}\text { CERCLA } \\
\text { PVC } \\
\text { RCRA } \\
\text { USGS }\end{array}$ & $\begin{array}{l}\text { Comprehensive } \mathrm{I} \\
\text { polyvinyl chlorid } \\
\text { Resource Conser } \\
\text { U.S. Geological }\end{array}$ & $\begin{array}{l}\text { ironmental I } \\
\text { ion and Rec } \\
\text { vey }\end{array}$ & $\begin{array}{l}\text { sponse, Compensation, and L } \\
\text { ery Act }\end{array}$ & ability Act & & & & \\
\hline
\end{tabular}




\subsection{Idaho National Laboratory Wells Decommissioned in CY 2010}

Table 11 indentifies 66 wells and boreholes that were abandoned (decommissioned). Several abandonment applications were submitted to the Idaho Department of Water Resources (IDWR) for review (Hutchison 2009; Hutchison 2010; Angle 2010) prior to abandoning the wells or boreholes. Well abandonments were overseen by an Idaho Cleanup Project professional engineer, as agreed to by the IDWR, to certify that all substantive requirements of State of Idaho well construction standards (IDAPA 37.03.09.025.16; IC 42-238) were met.

An evaluation of an INTEC CERCLA monitoring well, USGS-050, revealed that the original well installation may not have adequately isolated INTEC perched water zones to prevent subsurface leakage and downward movement of contaminated water. Extensive attempts to repair the well were unsuccessful; therefore, it was recommended that USGS-050 be abandoned. The Idaho Department of Environmental Quality and the Environmental Protection Agency were requested to approve abandoning this well (Butler 2007). Upon approval from the Department of Environmental Quality (Livieratos 2007) and the Environmental Protection Agency (Wilkening 2009), abandonment activity began on December 8, 2009, and was completed on February 17, 2010. Details and date of abandonment are shown in Table 11.

ICPP-MON-P-017 (MW-16), a perched CERCLA monitoring well at INTEC, was abandoned May 7, 2008. An abandonment application was submitted to IDWR in May, 2007 (McNeel 2007). This abandonment information had not been previously included in a Comprehensive Well Inventory update report. Details and date of the abandonment are shown in Table 11.

One well, SB-06, was not included in the previous abandonment applications because its status had previously been listed as "Abandoned." However, during a field check, it was discovered that the casing had not been sealed to the surface. This was remedied by adding casing seal (bentonite with water added) to the surface. This work was conducted in September 2009, but was not previously reported; therefore, it is included in this report as the record of its abandonment.

Twenty observation wells (TRA-A series) were drilled during the construction of the Material Test

Reactor/Experimental Test Reactor Warm Waste Ponds between 1962 and 1968. During the 2010 activity to abandon INL Site wells and boreholes, several of these observation wells could not be found during a field investigation. After researching historical documents, it was discovered that some had been destroyed during construction events, and some were abandoned as part of the remedial action for the Warm Waste Ponds in 1993 (MK-Ferguson of Idaho 1994). Details are listed in Table 11.

Ten boreholes and wells (Pit-9 series, TESTHOLE 0, W-02, and W-03) at RWMC also were discovered from field investigations to have been previously abandoned during the construction of the foundation for the Pit 9 building in 1994. Also, two other wells, W-01 and W-10, were discovered from field investigations to have been previously abandoned. Information concerning all of these wells and boreholes is included in this report to provide verification of their abandonment. The method and date of abandonment are shown in Table 11. Borehole PIT-9-P03 was abandoned in 2008 by filling the 3-inch casing with bentonite.

One instrumented borehole, TEM1-A, used to monitor CERCLA activities, that was not previously included in an abandonment application, is included in this report as a record of its abandonment. The borehole was used to monitor soil gas in the Subsurface Disposal Area. This borehole did not meet the definition of a well (IDAPA 37.03.09.010.66). However, it was included in a previous Comprehensive Well Inventory update. The status was inactive, and no future use was intended. Construction activities at Accelerated Retrieval Project VII required the immediate decommissioning of this borehole. The standard method of abandonment was followed. Details and date of abandonment are shown in Table 11. 
Appendix B contains maps showing the location of each well and borehole that was abandoned or determined to be abandoned in CY 2010 with the exception of TESTHOLE 0. Coordinates for the TESTHOLE 0 were not available. Also included in Appendix B, are diagrams, if available, that provide detailed construction and abandonment information on the abandoned wells and holes.

The CWI database maintains detailed well information that can be provided electronically to the state upon request. 
Table 11. Idaho National Laboratory boreholes and wells abandoned in Calendar Year 2010.

\begin{tabular}{|c|c|c|c|c|}
\hline \multicolumn{5}{|c|}{ INL WELLS ABANDONED IN CY 2010} \\
\hline Well Name & Well ID & Type & Status & Method and Date of Abandonment \\
\hline ICPP-MON-P-017 & 1072 & Monitoring & Abandoned & Filled 2-in. casing with bentonite on 5/7/2008. \\
\hline USGS-050 & 499 & Monitoring & Abandoned & $\begin{array}{l}\text { Pulled pump, filled } 4 \text {-in. casing and 6-in. open borehole with bentonite. Activity began } \\
12 / 8 / 09 \text { and completed } 2 / 17 / 10 \text {. }\end{array}$ \\
\hline LOFT-A01 & 209 & Observation & Abandoned & Filled 2-in. casing with bentonite on 6/7/2010. \\
\hline LOFT-A02 & 210 & Observation & Abandoned & Filled 2-in. casing with bentonite on 6/7/2010. \\
\hline LOFT-A03 & 211 & Observation & Abandoned & Filled 2-in. casing with bentonite on 6/7/2010. \\
\hline LOFT-A04 & 212 & Observation & Abandoned & Filled 2-in. casing with bentonite on 6/7/2010. \\
\hline LOFT-A05 & 213 & Observation & Abandoned & Filled 2-in. casing with bentonite on 6/7/2010. \\
\hline LOFT-A06 & 214 & Observation & Abandoned & Filled 2-in. casing with bentonite on 6/7/2010. \\
\hline LOFT-A07 & 215 & Observation & Abandoned & Filled 2-in. casing with bentonite on 6/7/2010. \\
\hline LOFT-A08 & 216 & Observation & Abandoned & Filled 2-in. casing with bentonite on 6/7/2010. \\
\hline LOFT-A09 & 217 & Observation & Abandoned & Filled 2-in. casing with bentonite on 6/7/2010. \\
\hline LOFT-A10 & 218 & Observation & Abandoned & Filled 2-in. casing with bentonite on 6/7/2010. \\
\hline LOFT-A11 & 219 & Observation & Abandoned & Filled 2-in. casing with bentonite on 6/7/2010. \\
\hline LOFT-A12 & 220 & Observation & Abandoned & Filled 2-in. casing with bentonite on 6/7/2010. \\
\hline LOFT-A13 & 221 & Observation & Abandoned & Filled 2-in. casing with bentonite on 6/7/2010. \\
\hline LOFT-A14 & 222 & Observation & Abandoned & Filled 2-in. casing with bentonite on 6/7/2010. \\
\hline LOFT-A15 & 223 & Observation & Abandoned & Filled 2-in. casing with bentonite on 6/7/2010. \\
\hline LOFT-A16 & 224 & Observation & Abandoned & Filled 2-in. casing with bentonite on 6/7/2010. \\
\hline LOFT-A17 & 225 & Observation & Abandoned & Filled 2-in. casing with bentonite on 6/7/2010. \\
\hline LOFT-A18 & 226 & Observation & Abandoned & Filled 2-in. casing with bentonite on 6/7/2010. \\
\hline LOFT-A19 & 227 & Observation & Abandoned & Filled 2-in. casing with bentonite on 6/7/2010. \\
\hline LOFT-A20 & 228 & Observation & Abandoned & Filled 2-in. casing with bentonite on 6/7/2010. \\
\hline SB-01 & 733 & Observation & Abandoned & Instruments removed, and 2-in. casing filled with bentonite on 7/13/10. \\
\hline SB-02 & 738 & Observation & Abandoned & Instrument line cut, and 2-in. casing filled with bentonite on 7/13/10. \\
\hline SB-03 & 739 & Observation & Abandoned & Filled 2-in. casing with bentonite on 7/13/10. \\
\hline SB-04 & 740 & Observation & Abandoned & Instrument line cut, and 2-in. casing filled with bentonite on 7/13/10. \\
\hline SB-05 & 741 & Observation & Abandoned & Filled 2-in. casing with bentonite on 7/14/10. \\
\hline
\end{tabular}


Table 11. (continued.)

\begin{tabular}{|c|c|c|c|c|}
\hline \multicolumn{5}{|c|}{ INL WELLS ABANDONED IN CY 2010} \\
\hline Well Name & Well ID & Type & Status & Method and Date of Abandonment \\
\hline SB-06 & 742 & Observation & Abandoned & Filled 6-in. casing with bentonite on 9/3/2009. \\
\hline SB-07 & 743 & Observation & Abandoned & Instrument removed and 2-in. casing filled with bentonite on 7/13/10. \\
\hline SB-08 & 744 & Observation & Abandoned & Filled 2-in. casing with bentonite on 7/14/10. \\
\hline TRA-06 & 808 & Monitoring & Abandoned & $\begin{array}{l}\text { Well was intended to be a monitoring well. Problems occurred during drilling and } \\
\text { replaced with TRA-06A. Was partially abandoned at that time, but not to surface. } \\
\text { Filled 6-in. casing with bentonite in August 2009. Not previously reported. }\end{array}$ \\
\hline TRA-A16 & 376 & Observation & Abandoned & $\begin{array}{l}\text { Abandoned as part of the remedial action for the Warm Waste Ponds. Verified } \\
5 / 20 / 2010 \text { (Jorgensen 2010a). }\end{array}$ \\
\hline TRA-A17 & 377 & Observation & Abandoned & $\begin{array}{l}\text { Abandoned as part of the remedial action for the Warm Waste Ponds. Verified } \\
5 / 20 / 2010 \text { (Jorgensen 2010a). }\end{array}$ \\
\hline TRA-A21 & 381 & Observation & Abandoned & $\begin{array}{l}\text { Abandoned as part of the remedial action for the Warm Waste Ponds. Verified } \\
5 / 20 / 2010 \text { (Jorgensen 2010a). }\end{array}$ \\
\hline TRA-A25 & 385 & Observation & Abandoned & $\begin{array}{l}\text { Abandoned as part of the remedial action for the Warm Waste Ponds. Verified } \\
5 / 20 / 2010 \text { (Jorgensen 2010a). }\end{array}$ \\
\hline TRA-A27 & 387 & Observation & Abandoned & Destroyed by construction in 1965 . Verified 6/20/2010 (Jorgensen 2010b). \\
\hline TRA-A28 & 388 & Observation & Abandoned & Filled 1.5-in. casing with bentonite on 7/13/10. \\
\hline TRA-A29 & 389 & Observation & Abandoned & Destroyed by construction in 1988. Verified 6/20/2010 (Jorgensen 2010b). \\
\hline TRA-A30 & 390 & Observation & Abandoned & Destroyed by construction after 1992. Verified 6/20/2010 (Jorgensen 2010b). \\
\hline TRA-A31 & 391 & Observation & Abandoned & Filled 1.6-in. casing with bentonite on 7/13/10. \\
\hline TRA-A33 & 393 & Observation & Abandoned & Destroyed by construction in 1990. Verified 6/20/2010 (Jorgensen 2010b). \\
\hline TRA-A34 & 394 & Observation & Abandoned & Destroyed by construction in 1975 . Verified 6/20/2010 (Jorgensen 2010b). \\
\hline TRA-A35 & 395 & Observation & Abandoned & Destroyed by construction in 1975. Verified 6/20/2010 (Jorgensen 2010b). \\
\hline TRA-A37 & 397 & Observation & Abandoned & $\begin{array}{l}\text { Abandoned as part of the remedial action for the Warm Waste Ponds. Verified } \\
6 / 20 / 2010 \text { (Jorgensen } 2010 \mathrm{~b} \text { ). }\end{array}$ \\
\hline TRA-A38 & 398 & Observation & Abandoned & $\begin{array}{l}\text { Abandoned as part of the remedial action for the Warm Waste Ponds. Verified } \\
5 / 20 / 2010 \text { (Jorgensen 2010a). }\end{array}$ \\
\hline TRA-A39 & 399 & Observation & Abandoned & $\begin{array}{l}\text { Abandoned as part of the remedial action for the Warm Waste Ponds. Verified } \\
5 / 20 / 2010 \text { (Jorgensen 2010a). }\end{array}$ \\
\hline TRA-A49 & 407 & Observation & Abandoned & Destroyed by construction in 1988 . Verified 5/20/2010 (Jorgensen 2010a). \\
\hline TRA-A51 & 409 & Observation & Abandoned & Destroyed by construction in 1977 . Verified 6/20/2010 (Jorgensen 2010b). \\
\hline
\end{tabular}


Table 11. (continued.)

\begin{tabular}{|c|c|c|c|c|}
\hline \multicolumn{5}{|c|}{ INL WELLS ABANDONED IN CY 2010} \\
\hline Well Name & Well ID & Type & Status & Method and Date of Abandonment \\
\hline TRA-A58 & 416 & Observation & Abandoned & $\begin{array}{l}\text { Abandoned as part of the remedial action for the Warm Waste Ponds. Verified } \\
6 / 20 / 2010 \text { (Jorgensen } 2010 \mathrm{~b} \text { ). }\end{array}$ \\
\hline TRA-A76 & 428 & Observation & Abandoned & Destroyed by construction after 1992. Verified 6/20/2010 (Jorgensen 2010b). \\
\hline TRA-A77 & 429 & Observation & Abandoned & Filled 2-in. casing with bentonite on 7/13/10. \\
\hline PIT-9-P01 & 841 & Borehole & Abandoned & $\begin{array}{l}\text { Field observation on } 7 / 12 / 10 \text {, revealed that well had been destroyed during the } \\
\text { construction of Pit } 9 \text { building in } 1994 \text {. }\end{array}$ \\
\hline PIT-9-P02 & 842 & Borehole & Abandoned & $\begin{array}{l}\text { Field observation on } 7 / 12 / 10 \text {, revealed that well had been destroyed during the } \\
\text { construction of Pit } 9 \text { building in } 1994 \text {. }\end{array}$ \\
\hline PIT-9-P03 & 843 & Borehole & Abandoned & Filled 3-inch casing with bentonite 8/13/2008. \\
\hline PIT-9-P04 & 844 & Borehole & Abandoned & $\begin{array}{l}\text { Field observation on } 7 / 12 / 10 \text {, revealed that well had been destroyed during the } \\
\text { construction of Pit } 9 \text { building in } 1994 \text {. }\end{array}$ \\
\hline PIT-9-P05 & 845 & Borehole & Abandoned & $\begin{array}{l}\text { Field observation on } 7 / 12 / 10 \text {, revealed that well had been destroyed during the } \\
\text { construction of Pit } 9 \text { building in } 1994 \text {. }\end{array}$ \\
\hline PIT-9-P06 & 846 & Borehole & Abandoned & $\begin{array}{l}\text { Field observation on } 7 / 12 / 10 \text {, revealed that well had been destroyed during the } \\
\text { construction of Pit } 9 \text { building in } 1994 \text {. }\end{array}$ \\
\hline PIT-9-P07 & 847 & Borehole & Abandoned & $\begin{array}{l}\text { Field observation on } 7 / 12 / 10 \text {, revealed that well had been destroyed during the } \\
\text { construction of Pit } 9 \text { building in } 1994 \text {. }\end{array}$ \\
\hline PIT-9-P08 & 848 & Borehole & Abandoned & $\begin{array}{l}\text { Field observation on } 7 / 12 / 10 \text {, revealed that well had been destroyed during the } \\
\text { construction of Pit } 9 \text { building in } 1994 \text {. }\end{array}$ \\
\hline TESTHOLE 0 & 350 & None & Abandoned & $\begin{array}{l}\text { Field observation on } 7 / 12 / 10 \text {, could not locate well. Believed to be previously } \\
\text { abandoned by filling borehole with native soil. }\end{array}$ \\
\hline $\mathrm{W}-01$ & 874 & Scientific instrumentation & Abandoned & Field observation on $7 / 12 / 10$, revealed that well had been previously abandoned. \\
\hline $\mathrm{W}-02$ & 875 & Scientific instrumentation & Abandoned & $\begin{array}{l}\text { Field observation on } 7 / 12 / 10 \text {, revealed that well had been covered over with the } \\
\text { foundation of the PIT } 9 \text { building in } 1994 \text {. }\end{array}$ \\
\hline $\mathrm{W}-03$ & 576 & Scientific instrumentation & Abandoned & $\begin{array}{l}\text { Field observation on } 7 / 12 / 10 \text {, revealed that well had been covered over with the } \\
\text { foundation of the PIT } 9 \text { building in } 1994 \text {. }\end{array}$ \\
\hline $\mathrm{W}-10$ & 582 & Scientific instrumentation & Abandoned & Field observation on $7 / 12 / 10$, revealed that well had been previously abandoned. \\
\hline TEM1-A & 863 & Vapor port & Abandoned & Instruments lines were capped and 4.5-in. casing filled with bentonite on 10/20/10. \\
\hline BORAX & 868 & Storm water injection well & Abandoned & Casing cut off $10 \mathrm{in}$. bls then filled with $1.2 \mathrm{yd}^{3}$ of bentonite on $11 / 04 / 10$. \\
\hline
\end{tabular}




\section{REFERENCES}

Angle, Bruce M., Acting Director, Environmental Support and Services, INL, to M. Anders, IDWR, September 9, 2010, "Submittal of the Authorization to Abandon a Well form for the Decommissioning of BORAX Well and the Technical Requirements for Decommissioning BORAX Well ID 868," CCN 221981.

Butler, J. Lane, Area Project Manager, Environmental Restoration, ICP, to N. Ceto, EPA and D. Koch, Idaho DEQ, May, 7, 2007, "Request to Plug Abandon INTEC Monitoring Wells," CCN 304830.

Department of Justice, Environment and Natural Resources Division, 1990, Water Rights Agreement between the State of Idaho and the United States, for the United States Department of Energy.

District Court-SRBA, Twin Falls Co., Idaho, 2003, Order of Partial Decree for Water Right 34-10901 (United States Department of Energy, Idaho National Engineering and Environmental Laboratory), Case No. 39576, June 20, 2003.

DOE-ID, 2009, HWMA/RCRA Landfill Closure Plan for the CPP-601/627/640 Landfill, DOE/ID-11408, Rev 1, U. S. Department of Energy, Idaho Operations Office, December 2009.

DOE-ID, 2010, Completion Summary for Well NRF-16 near the Naval Reactors Facility, Idaho National Laboratory, Idaho, DOE/ID-22210, U. S. Department of Energy.

Hutchison, D. P., Director, Environmental and Regulatory Services, ICP, to D. Dunn, IDWR, July 21, 2009, "Wells for Potential Decommissioning (Abandonment) at the Idaho National Laboratory Site," CCN 308819.

Hutchison, D. P., Director, Environmental and Regulatory Services, ICP, to D. Dunn, IDWR, August 18, 2010, "Decommissioning Notification for Fifty-Two Wells at the Idaho National Laboratory Site," CCN 310639.

IC 42-238, 2010, "Well Drillers' Licenses and Operator Permits," Idaho Code.

IDAPA 37.03.09.025.16, 2009, "Decommissioning (Abandoning) of Wells,” Idaho Administrative Procedures Act.

IDAPA 37.03.09.010.66, 2009, “Well,” Idaho Administrative Procedures Act.

Jorgensen, M. D., ER CERCLA Field Activities, ICP, to W. Jolley and R. Bowser, May 20, 2010a, "Well Abandonment Walkdown," CCN 310366.

Jorgensen, M. D., ER CERCLA Field Activities, ICP, to R. Bowser and W. Jolley, June 10, 2010b, “TRA Wells", CCN 310367.

Livieratos, T., WAG 3 Project Manager, DEQ, to J. L. Butler, ER, ICP, May, 16, 2007, "Request to Plug Abandon INTEC Monitoring Wells", CCN 304922.

McNeel, K,. Director, Environmental and Regulatory Services, ICP, to D. Dunn, IDWR, May 2, 2007, "Application for Authorization to Abandon Wells at the Idaho National Laboratory Site," CCN 304727 and CCN 306009.

MK-Ferguson of Idaho, 1994, Remedial Acton Report, Test Reactor Area Warm Waste Pond Interim Action, Operable Unit 2-10, Document Number: 02.010.2.1.209.01, INEL Environmental Restoration, MK-Ferguson of Idaho, June 1994.

Street, L. V., INEEL, to D. Dunn, IDWR, September 4, 2001, "INEEL Comprehensive Well Surveys and Annual Water Use Reports," CCN 25370.

Wilkening, M, EPA, to N. Hernandez, DOE-ID, October 14, 2009, "USGS 50 Well Abandonment Plan," CCN 311104. 
Appendix A

\section{Maps and Construction Diagrams for New and Modified Wells}


